\title{
A nurse led clinic and computer decision support software for anticoagulation decisions were as effective as a hospital clinic
}

\author{
Fitzmaurice DA, Hobbs FD, Murray ET, et al. Oral anticoagulation management in primary care with the use of \\ computerized decision support and near-patient testing. A randomized, controlled trial. Arch Intern Med 2000 Aug \\ 14/28;160:2343-8.
}

QUESTION: For patients who require oral anticoagulation, is a primary care, nurse led clinic that uses on site testing and computerised decision support software more effective than routine hospital care for maintaining appropriate international normalised ratios (INRs)?

\section{Design}

Randomised (unclear allocation concealment*), unblinded, ${ }^{*}$ controlled trial, stratified by practice size, with 1 year follow up.

\section{Setting}

12 of 21 potential general practices and 3 hospital clinics in Birmingham, UK.

\section{Patients}

224 adult patients ( $55 \%$ men) who were receiving warfarin. Follow up was $82 \%$.

\section{Intervention}

122 patients were allocated to nurse led management in a primary care setting. The nurse met with the patient, measured the INR with on site equipment, and used the computer program (Anticoagulation Management Support System [Softop Information Systems, Warwick, England, UK]) to direct dosing decisions. The program was based on the British Society of Haematology guidelines and had 2 main ranges of INR (2.0 to 3.0 and 3.0 to 4.5). If a dosing change was suggested, the nurse verified the change with medical staff at the clinic. 102 patients were allocated to hospital based care (2 of the 3 sites were staffed by a physician during the study, and 1 site started with physician supervision before switching to a technician and another computer decision support program.)

\section{Main outcome measures}

Proportion of patients who achieved appropriate INR control and mean percentage of time each patient spent within a therapeutic range of INR.

\section{Main results}

8 patients in the nurse led group returned to hospital clinic care. Patients in the nurse led group had a higher percentage of time spent in the therapeutic INR range than patients in the hospital group $(69 \%$ v $57 \%$, $\mathrm{p}<0.001)$. The groups did not differ for the proportion of tests in therapeutic range (although both groups improved from baseline levels), all cause mortality (3 deaths in each group), serious adverse events (3 in each group), or overall adverse events. The mean intervention cost per patient was US $\$ 270$ in the nurse led group and $\$ 110$ in the hospital group.

\section{Conclusion}

A nurse led clinic in the primary care setting that included on site blood tests and a computer decision support program for patients who were receiving warfarin was at least as effective as a hospital based clinic.

*See glossary.

\section{COMMENTARY} primary care setting with improved overall continuity of care. toring and self management. ${ }^{3}$ munities. Arch Intern Med 2000;160:967-73.

\begin{abstract}
Sources of funding: $U K$ Medical Research Council Reaching Our Potential Award scheme and UK National Health Service Research and Development Primary and Secondary Care Interface Programme.
\end{abstract}

For correspondence: Dr D A Fitzmaurice, Department of General Practice, Medical

School, University of Birmingham, Birmingham B15 2TT, UK. Fax $+44(0) 121$ 4143759 .

A modified version of this abstract also appears in Evidence-Based Nursing.

The growing number of patients receiving long term oral anticoagulation has created a substantial primary care management burden. To achieve benefit-risk ratios similar to those reported in randomised controlled trials, patients' INRs must be maintained in a narrow therapeutic range. However, primary care providers have difficulty in replicating the success observed in the trial setting. Approximately $50 \%$ of the time, patients who are receiving anticoagulants are not within the therapeutic range. ${ }^{1}$ For patients with atrial fibrillation, the combined rate of major bleeding complications and stroke is 2 to 3 times higher in the "usual" care setting than in special anticoagulation clinics. ${ }^{2}$ Anticoagulation management clinics are more likely than usual care settings to achieve optimal anticoagulation because of dedicated providers, appropriate follow up, access to INR monitoring, decision support systems, and patient education. ${ }^{2}{ }^{3}$ Specialised ambulatory anticoagulation management services may be provided in a hospital environment and are often delivered by non-physician providers or pharmacists who are directed by a physician who has no responsibility for the primary care of the patients. With appropriate restructuring, optimal long term anticoagulation may be achieved within a

The stratified randomised controlled trial by Fitzmaurice $e t$ al shows the effectiveness of primary care anticoagulation management that is delegated to a nurse with access to on site INR testing and computerised decision support. Consequently, this study supports the notion that the special features of anticoagulation management services can be incorporated into primary care. ${ }^{2}$ Promising strategies include patient self moni-

Brian P Schmitt, MD Northwestern University Medical School Chicago, Illinois, USA

1 Samsa GP, Matchar DB, Goldstein LB, et al. Quality of anticoagulation management among patients with atrial fibrillation: results of a review of medical records from 2 com-

2 Ansell JE. The quality of anticoagulation management. Arch Intern Med 2000;160:895-6.

3 Ansell JE, Hughes R. Evolving models of warfarin management: anticoagulation clinics, patient self-monitoring, and patient self-management. Am Heart J 1996;132:1095-100. 